
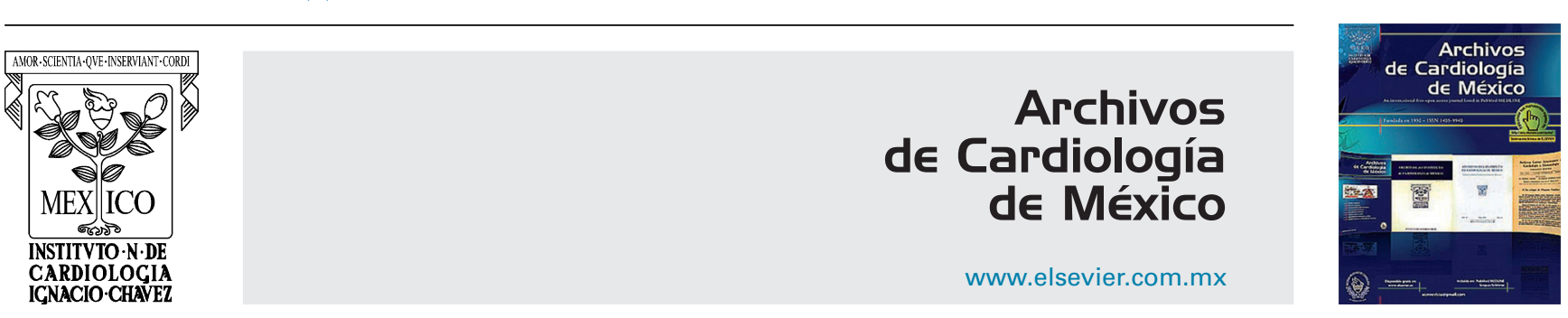

INVESTIGACIÓN CLÍNICA

\title{
Efecto agudo del consumo de yerba mate (llex paraguariensis) sobre el ritmo cardíaco en pacientes derivados para estudio Holter
}

\author{
Alejandro Cuesta ${ }^{a, *}$, Carina Guigou $^{a}$, Analía Varela ${ }^{a}$, Lorena Ferrero ${ }^{b}$, \\ María Clara Charlin ${ }^{a}$ y Ricardo Lluberas ${ }^{a}$
}

\begin{abstract}
a Servicio de Cardiología, Departamento Clínico de Medicina, Hospital de Clínicas, Facultad de Medicina, Universidad de la República, Montevideo, Uruguay

b Servicio de Cardiología, Hospital Central de las Fuerzas Armadas, Montevideo, Uruguay
\end{abstract}

Recibido el 6 de marzo de 2018; aceptado el 1 de mayo de 2018

\section{PALABRAS CLAVE \\ Ilex paraguariensis; Yerba mate; Arritmias; Extrasístoles supraventriculares; Uruguay}

\begin{abstract}
Resumen
Introducción: El consumo de yerba mate (YM) (Ilex paraguariensis) es común en Sudamérica y se extiende a todo el mundo. Contiene muchas sustancias bioactivas que pueden ser beneficiosas. También existe la opinión de que puede tener efectos nocivos sobre el ritmo cardíaco, pero no encontramos investigaciones al respecto.

Objetivo: Nuestro objetivo fue evaluar el efecto agudo de YM sobre el ritmo, en pacientes enviados para realizar un Holter.

Método: Realizamos un estudio clínico seudoexperimental, de tipo antes y después. Tomamos 50 pacientes consumidores habituales de YM y se les realizó un Holter tomando YM y otro luego de 24 sin consumir. También se controló el uso de otros productos que pudieran interferir.

Resultados: El 52\% fueron mujeres y la edad, de $55 \pm 15$ años. El 78\% tenía al menos un factor de riesgo vascular, el $44 \%$ cardiopatía estructural y el $90 \%$ tomaba medicación cardiovascular. La frecuencia cardíaca (FC) con y sin YM fue similar. Solo la FC mínima horaria fue menor tomando YM $(61 \pm 10$ vs. $63 \pm 9, p=0.021)$. No hubo diferencia significativa en la incidencia de arritmias sostenidas, no sostenidas ni en la extrasistolia ventricular. El total de extrasístoles supraventriculares fue mayor sin YM $(952 \pm 3,538$ vs. $1,294 \pm 5,201, p=0.014)$ y la cantidad máxima horaria también $(86 \pm 302$ vs. $107 \pm 360, p=0.032)$. No hubo diferencia en la variabilidad de la FC.
\end{abstract}

\footnotetext{
* Autor para correspondencia. Cairoli 2026, CP 11600, Montevideo (Uruguay). Teléfono: +59899617427.

Correo electrónico: arritmia@yahoo.com (A. Cuesta).
} 


\section{KEYWORDS}

Ilex paraguariensis;

Yerba mate;

Arrhythmias;

Supraventricular

extrasystoles;

Uruguay

Conclusiones: En pacientes cardiológicos tomadores de YM, su consumo de la forma habitual no se asoció con cambios importantes de la FC ni con un aumento en la incidencia de arritmias. Hubo menos actividad ectópica supraventricular.

( 2018 Instituto Nacional de Cardiología Ignacio Chávez. Publicado por Masson Doyma México S.A. Este es un artículo Open Access bajo la licencia CC BY-NC-ND (http://creativecommons. org/licenses/by-nc-nd/4.0/).

Acute effect of yerba mate (Ilex paraguariensis) consumption on heart rhythm in patients referred for Holter study

\begin{abstract}
Introduction: The consumption of yerba mate (YM) (Ilex paraguariensis) is common in South America and is now used all over the world. It contains many bioactive substances that can be beneficial. There is also the opinion that it may have harmful effects on heart rhythm, but no studies have been found on this.

Objective: The aim of this study was to evaluate the acute effect of YM on heart rhythm in patients referred for a Holter study.

Method: A before and after pseudo-experimental clinical study was conducted by performing a Holter on 50 habitual users of YM, and another one after 24 without YM consumption. The use of other products that could interfere was also controlled.

Results: The mean age of the subjects was $55 \pm 15$ years, and $52 \%$ were women. At least one vascular risk factor was found in $78 \%$, with $44 \%$ structural heart disease, and $90 \%$ taking cardiovascular medication. The heart rate $(\mathrm{HR})$ with and without $\mathrm{YM}$ was similar. Only the minimum hourly HR was lower when taking YM $(61 \pm 10$ vs. $63 \pm 9, P=.021)$. There was no significant difference in the incidence of sustained or non-sustained arrhythmias, or in ventricular extrasystoles. The total number of supra-ventricular premature beats was higher without YM $(952 \pm 3,538$ vs. $1,294 \pm 5,201, P=.014)$ and also the maximum hourly ( $86 \pm 302$ vs. $107 \pm 360, P=.032$ ). There was no difference in HR variability.

Conclusions: In cardiology patients, usual users of YM, habitual consumption was not associated with significant changes in HR or an increase in the incidence of arrhythmias. There was less ectopic supraventricular activity.

( 2018 Instituto Nacional de Cardiología Ignacio Chávez. Published by Masson Doyma México S.A. This is an open access article under the CC BY-NC-ND license (http://creativecommons. org/licenses/by-nc-nd/4.0/).
\end{abstract}

\section{Introducción}

El consumo de yerba mate (YM) (Ilex paraguariensis) en infusión es una práctica muy común en Sudamérica. Debido a las migraciones y también por la aparición de bebidas «energéticas» elaboradas con base en la misma planta se está extendiendo a todo el mundo. En los países de la cuenca del río de la Plata tiene un rol importante en la alimentación y es parte de la cultura e identidad regional. Como ejemplo, el $85 \%$ de la población adulta en Uruguay consume YM regularmente ${ }^{1}$.

La YM contiene muchas sustancias bioactivas: compuestos polifenólicos, metilxantinas (teofilina, teobromina, cafeína), derivados cafeólicos, saponinas triterpénicas y múltiples minerales básicos del metabolismo ${ }^{2,3}$. Estas sustancias explicarían algunas de las propiedades benéficas que se han adjudicado a la infusión: capacidad antioxidante, efecto hipolipidemiante, reducción de la obesidad, inhibición de la ateroesclerosis, vasodilatación periférica, protección antiisquémica y beneficios a nivel del sistema nervioso central ${ }^{4-11}$.

Al igual que con otras infusiones como el té y el café, existe preocupación por su rol en algunos tipos de cáncer, particularmente de las vías digestivas y urinarias. Pero es difícil de precisar, en la medida en que se confunde con su asociación al tabaquismo y al consumo de agua con alta temperatura ${ }^{12}$.

Existe la opinión extendida de que el consumo de YM puede tener efectos nocivos sobre el ritmo cardíaco, favoreciendo la ocurrencia de arritmias rápidas. Es habitual que miembros del equipo de salud contraindiquen su uso en pacientes con cardiopatías. Esto es una modificación importante de su alimentación y hábitos y afecta su calidad de vida. También podríamos estar privándolos de los eventuales efectos beneficiosos. No encontramos en la bibliografía estudios que avalen esta recomendación y nos pareció importante comenzar a investigarlo. 


\section{Objetivo}

Evaluar el efecto agudo del consumo de YM sobre el ritmo cardíaco en pacientes enviados para realizar un estudio Holter por enfermedad cardiovascular. Tenemos especial interés en buscar el efecto clínico en pacientes con afección cardíaca. En ellos se genera más incertidumbre sobre proscribir o no el consumo de YM.

\section{Material y métodos}

Realizamos un estudio clínico seudoexperimental de tipo antes y después. Tomamos las mediciones en el mismo grupo de personas, expuestas y no expuestas a la toma habitual de YM.

\section{Pacientes}

A todos los pacientes enviados a los servicios de Holter de los 2 centros participantes y que eran consumidores habituales de YM se les propuso participar en el estudio hasta que alcanzamos la cifra de 50 incluidos que cumplieron el protocolo. Eran todos sujetos enviados por sus cardiólogos tratantes. El Holter estaba indicado por la razón que ellos consideraron. Los tratantes no participaron en este trabajo y no se indicaron estudios solo con este fin. Se excluyeron los pacientes que tenían puesto un marcapasos y que tenían indicada alguna medicación con xantinas. Cuando el paciente se presentó para realizar el Holter se le explicaron las características de la investigación. Se le entregó un instructivo y medio kilo de YM envasada para garantizar la disponibilidad del producto. El paciente tuvo la libertad de utilizar cualquier marca comercial de YM. Este estudio no tuvo el apoyo de ninguna empresa del ramo. Se realizó con los fondos e infraestructura de los centros que participaron.

\section{Consentimiento}

El protocolo de investigación fue realizado en consonancia con la Declaración de Helsinki y aprobado por nuestro Comité de Ética. La inclusión en el estudio fue individual y voluntaria. A todos los pacientes se les solicitó el consentimiento informado, que podían suspender cuando quisieran. Se garantizó la confidencialidad de la información.

\section{Procedimiento}

A cada paciente incluido se le realizaron 2 Holter de $24 \mathrm{~h}$. Para la realización y el análisis de los Holter se utilizaron los mismos equipos y programas (RhythmScan 8800 Holter 1992-7, PHCS Inc.). Durante el primer estudio se le pidió al paciente que consumiera YM en su forma habitual. Para el segundo estudio, considerando la vida media de las sustancias contenidas, se le pidió que desde el día anterior y el mismo día no consumiera YM. Se les pidió también que durante ninguno de los 2 registros consumieran otra bebida o alimento que contuviera cafeína u otra xantina; tampoco medicación. Para ello se le proporcionó una lista de los productos y medicamentos, a su eventual alcance, que las contuvieran. En el instructivo había también números de teléfono para aclarar dudas en cualquier momento. Se insistió en que durante ambos Holter el paciente tomara la misma mediación prescrita (fármaco, presentación, dosis y posología) por su médico tratante y realizara una actividad física e intelectual similar a la habitual. Al retirar el segundo Holter se interrogó al paciente sobre el cumplimiento de lo indicado, aclarándole que lo contrario no tenía ninguna consecuencia.

\section{Variables}

Se registraron las variables demográficas, la indicación del Holter, el tipo de cardiopatía, la medicación y las variables para cuantificar la cantidad de YM consumida. Cada Holter fue descargado e informado con rigurosidad por el técnico y los médicos participantes en el estudio.

\section{Análisis estadístico}

Se realizó utilizando el paquete estadístico SPSS. Las variables continuas se expresan como media $\pm D E$. Para su comparación se utilizó el test de la t para muestras pareadas y para las variables no paramétricas se usó el test de Wilcoxon para muestras pareadas. Las variables nominales se describen mediante frecuencia absoluta y relativa y se compararon con el test de McNemar. Se consideró significativa una $p<0.05$.

\section{Resultados}

Incluimos 50 pacientes a los que se realizaron 2 estudios Holter en las condiciones exigidas en el protocolo. De los pacientes que dieron su consentimiento e ingresaron no se incluyeron 5 por algún incumplimiento.

En la tabla 1 mostramos las características del grupo de estudio. El número de pacientes provenientes de cada centro fue similar. El $52 \%$ fueron mujeres y la edad, de $55 \pm 15$ años. A la mayoría de los pacientes les habían indicado un Holter para estudiar el síntoma de palpitaciones (42\%). Hubo 8 pacientes enviados para el estudio de fibrilación auricular (FA) y 3 por síncope. La gran mayoría (78\%) tenía al menos un factor de riesgo vascular mayor modificable y un $44 \%$ tenían una cardiopatía estructural ya conocida.

En 46 pacientes (92\%) se contaba con un ecocardiograma de menos de un año. Había 12 pacientes con una valvulopatía al menos moderada y 17 con una función sistólica del ventrículo izquierdo menor al $50 \%$. El $90 \%$ de los pacientes tomaba al menos una medicación cardiovascular (tabla 1).

Todos los pacientes tomaron mate el día del primer estudio. El $80 \%$ preparó la infusión 2 o más veces en el día y el $92 \%$ se sirvieron 10 o más veces. Con estos datos estimamos un consumo medio de $500 \mathrm{ml}$ de infusión/día.

En la tabla 2 mostramos los valores de las variables del Holter con y sin mate comparadas. El tiempo medio de registro en ambas situaciones fue similar.

La frecuencia cardíaca (FC) media con y sin YM fue la misma. La FC mínima y la FC máxima (con base en 4 ciclos) también fueron similares. Solo la FC mínima horaria resultó algo menor en el Holter hecho con YM (61 \pm 10 vs. $63 \pm 9$, $\mathrm{p}=0.021$, IC 95\% $1.5[0.2-2.8]$ ). 
Tabla 1 Descripción de las características de todos los pacientes incluidos, su enfermedad cardiovascular, medicación y la indicación del estudio Holter

\begin{tabular}{|c|c|}
\hline Total, $n(\%)$ & $50(100)$ \\
\hline Hospital de Clínicas & $26(52)$ \\
\hline Hospital Militar & $24(48)$ \\
\hline Mujer, $n(\%)$ & $26(52)$ \\
\hline Edad (años), media $\pm D E$ & $55 \pm 15$ \\
\hline \multicolumn{2}{|l|}{ Indicación del Holter, n (\%) } \\
\hline Palpitaciones & $21(42)$ \\
\hline Fibrilación auricular & $8(16)$ \\
\hline Extrasistolia & $5(10)$ \\
\hline Síncope & $3(6)$ \\
\hline Otras & $13(26)$ \\
\hline Factores de riesgo $\mathrm{CV}, \mathrm{n}(\%)$ & $39(78)$ \\
\hline Hipertensión arterial & $32(64)$ \\
\hline Diabetes & $6(12)$ \\
\hline Dislipidemia & $18(36)$ \\
\hline Fumador & $13(26)$ \\
\hline Cardiopatía estructural, n (\%) & $22(44)$ \\
\hline Valvular & $5(10)$ \\
\hline Dilatada & $8(16)$ \\
\hline Hipertensiva & $10(10)$ \\
\hline Isquémica & $7(14)$ \\
\hline \multicolumn{2}{|l|}{ Ecocardiograma } \\
\hline FEVI (\%), media $\pm \mathrm{DE}$ & $52 \pm 11$ \\
\hline Diámetro de $\mathrm{Al}(\mathrm{mm})$, media $\pm \mathrm{DE}$ & $38 \pm 6$ \\
\hline Estenosis aórtica, n (\%) & $3(6)$ \\
\hline Insuficiencia aórtica, n (\%) & $1(2)$ \\
\hline Estenosis mitral, n (\%) & $1(2)$ \\
\hline Insuficiencia mitral, n (\%) & $7(14)$ \\
\hline Medicación, n (\%) & $45(90)$ \\
\hline IECA & $30(60)$ \\
\hline Betabloqueante & $21(42)$ \\
\hline Estatina & $14(28)$ \\
\hline Antagonista del calcio & $8(16)$ \\
\hline Amiodarona & $3(6)$ \\
\hline Digoxina & $2(4)$ \\
\hline Flecainida & $1(2)$ \\
\hline
\end{tabular}

Al: aurícula izquierda; CV: cardiovascular; DE: desviación estándar; FEVI: fracción de eyección del ventrículo izquierdo; IECA: inhibidor de la enzima convertidora de angiotensina.

Hubo un poco más de extrasístoles ventriculares con YM, pero la diferencia no fue significativa. En 23 pacientes el número de extrasístoles ventriculares fue mayor tomando YM, en 15 fue menor y en 12 fue el mismo (tabla 2).

No hubo episodios de taquicardia ventricular (TV) sostenida. Hubo 2 pacientes que tuvieron episodios de TV no sostenida (definida como 3 o más latidos de origen ventricular) con y sin YM. Hubo 3 pacientes con episodios de TV no sostenida solo con YM y 2 solo si YM.

Hubo menos extrasístoles supraventriculares (ESV) tomando YM. Las diferencias para el total de ESV en el día y la cantidad máxima de ESV/h fueron significativas (tabla 2). La cantidad de episodios de taquicardia supraventricular fue la mitad tomando YM, pero esta diferencia no alcanzó significación estadística (tabla 2). En 19 pacientes la ESV fue menor tomando mate, en 24 fue la misma y en 7 fue mayor.

No hubo diferencias en los 2 indicadores de variabilidad de FC considerados.

\section{Fibrilación auricular}

Incluimos 8 pacientes con antecedente de FA: 4 permanente y 4 paroxística. En ninguno de los pacientes con FA paroxística se registró la arritmia con o sin YM. En 2 pacientes sin antecedentes de FA se registró un episodio de FA paroxística: uno con YM y en otro sin YM. En los 4 pacientes con FA permanente no hubo diferencias en el control de la FC con y sin mate.

La ocurrencia de alteraciones de la conducción auriculoventricular y pausas mayores de $3 \mathrm{~s}$ fue mínima en todo el grupo, para ambas situaciones, y no se analizó. No encontramos diferencias entre géneros y por el tamaño de la muestra no hicimos otros subanálisis.

\section{Discusión}

El efecto clínico humano de un alimento es siempre difícil de evaluar por varios motivos. Los alimentos suelen tener efectos pequeños a corto plazo, difíciles de objetivar en una variable biológica. Contienen múltiples moléculas biológicamente activas, en cantidades absolutas y relativas que varían mucho. Las personas consumen un alimento en porciones variables, en distintos momentos del día y con distinta preparación. En el caso de la YM se agrega la forma de preparación y la temperatura del agua. Por otro lado, los estudios de intervención que administran un principio activo aislado de un alimento y observan un efecto biológico no necesariamente reproducen el resultado del alimento entero. Nosotros estudiamos el efecto de la YM en la forma habitual de consumo en nuestro país.

Estudiamos a un grupo de pacientes derivados por su cardiólogo, la mayoría con factores de riesgo cardiovascular, muchos con cardiopatía de base, alteraciones del ritmo y/o medicación cardiovascular (tabla 1). Eran todos pacientes estables y sin grados avanzados de su enfermedad. Este perfil de paciente es muy frecuente en la población que se asiste en cardiología, pero no representa a pacientes en situaciones clínicas más complejas.

No encontramos un efecto significativo en la FC a lo largo del día. La diferencia en la FC mínima horaria fue significativa, pero de una magnitud pequeña (tabla 2). Nuestros hallazgos alejan la posibilidad de algún efecto biológico importante sobre la FC y se inclinan hacia una acción neutra sobre el conjunto del automatismo cardíaco y los sistemas de regulación. Tampoco hubo diferencias en los indicadores de variabilidad considerados.

No hubo episodios de TV sostenida ni diferencias en la ocurrencia de TV no sostenidas. Tampoco en la ocurrencia de FA ni otras taquicardias supraventriculares sostenidas. La incidencia de todas estas fue muy baja.

Hubo más cantidad de ESV en el día sin YM. La cantidad máxima de ESV en una hora también fue mayor sin YM. Las dispersiones de estas variables son grandes y ello relativiza el hallazgo, pero fue claro que en muchos más pacientes aparecieron o aumentaron las ESV el día que no tomaron 
Tabla 2 Valores de las variables consideradas del Holter, con y sin consumo de yerba mate y comparadas

\begin{tabular}{|c|c|c|c|}
\hline & Con YM & Sin YM & $\mathrm{p}$ \\
\hline Tiempo de registro (min), media $\pm D E$ & $1,376 \pm 76$ & $1,385 \pm 58$ & NS \\
\hline FC mínima (cpm), media $\pm D E$ & $52 \pm 10$ & $52 \pm 9$ & NS \\
\hline FC máxima (cpm,) media $\pm D E$ & $127 \pm 23$ & $128 \pm 25$ & NS \\
\hline$F C$ media $(c p m)$, media $\pm D E$ & $77 \pm 12$ & $78 \pm 10$ & NS \\
\hline$F C$ horaria mínima $(c p m)$, media $\pm D E$ & $61 \pm 10$ & $63 \pm 9$ & 0.021 \\
\hline FC horaria máxima (cpm), media $\pm D E$ & $98 \pm 17$ & $101 \pm 18$ & NS \\
\hline TV no sostenida, $n$ (\%) & $5(10)$ & $4(8)$ & NS \\
\hline \multicolumn{4}{|l|}{ Extrasistolia ventricular $(n)$, media $\pm D E$} \\
\hline Totales & $417 \pm 860$ & $322 \pm 597$ & NS \\
\hline Cantidad máxima horaria & $73 \pm 141$ & $58 \pm 109$ & NS \\
\hline Cantidad de duplas & $3 \pm 15$ & $2 \pm 9$ & NS \\
\hline \multicolumn{4}{|c|}{ Extrasistolia supraventricular (n), media $\pm D E$} \\
\hline Totales & $952 \pm 3,538$ & $1,294 \pm 5,201$ & 0.014 \\
\hline Cantidad máxima horaria & $86 \pm 302$ & $107 \pm 360$ & 0.032 \\
\hline Rachas supraventriculares & $43 \pm 262$ & $84 \pm 565$ & NS \\
\hline Total de extrasístoles (n), media $\pm D E$ & $1,369 \pm 3,568$ & $1,616 \pm 5,202$ & NS \\
\hline$S D N N$, media $\pm D E$ & $119 \pm 39$ & $114 \pm 38$ & NS \\
\hline$R M S S$, media $\pm D E$ & $38 \pm 23$ & $37 \pm 23$ & NS \\
\hline
\end{tabular}

DE: desviación estándar; FC: frecuencia cardíaca; NS: no significativo; RMSS: raíz cuadrada de las diferencias al cuadrado de intervalos; SDNN: desvío estándar de intervalos QRS normal a normal; TV: taquicardia ventricular; YM: yerba mate.

mate. Habrá que seguir investigando la hipótesis de que la YM tiene algún efecto protector o que la abstinencia tiene este efecto adverso.

No hay posibilidades reales de realizar una comparación con placebo. Lograr un grupo importante de pacientes no tomadores de YM que accedan a hacerlo para un estudio ha fallado. Podríamos realizar un estudio de casos y controles pareados, ajustados por las posibles variables de confusión, particularmente la presencia, el tipo y el grado de cardiopatía. Estamos en camino de definir esas variables, pero es dudoso que tenga más valor que el presente diseño.

Los síntomas como las palpitaciones son un indicador muy pobre. Los efectos de la cafeína sobre el sistema nervioso central, y particularmente sobre la vigilia, pueden explicar la mayoría de ellos ${ }^{13}$.

El café comparte varios compuestos con la YM y hay mucha más información sobre él. También existía el planteo de que podía tener efectos cardiovasculares deletéreos, pero con las investigaciones se ha ido abandonando. Hay estudios epidemiológicos que han mostrado una relación inversa entre la mortalidad total y la cardiovascular con el consumo de café ${ }^{14-16}$. No se pudo demostrar que la cafeína era proarrítmica en sujetos sanos ${ }^{17,18}$. Incluso se encontró que el consumo de café puede tener un efecto protector respecto a la $\mathrm{FA}^{19,20}$.

Nuestros hallazgos cuestionan la idea de que el consumo de YM es proarrítmico. Restan argumentos para contraindicar su consumo en personas que lo toman habitualmente y permite plantear hipótesis en el sentido contrario.

\section{Conclusiones}

En pacientes controlados en un servicio de cardiología y tomadores de $Y M$, su consumo de la forma habitual no se asoció con cambios importantes de la FC. Tampoco se evidenció que el consumo favoreciera de forma aguda la aparición de alteraciones del ritmo. La única diferencia significativa encontrada fue una menor ocurrencia de actividad ectópica supraventricular con YM, que habrá que continuar estudiando para poder corroborarla.

\section{Limitaciones}

Este estudio analizó el efecto agudo del consumo de YM en personas que la toman habitualmente. No se puede inferir nada de su efecto a largo plazo y/o en personas no consumidoras. No realizamos dosificaciones en sangre de principios activos, ya que esto no era necesario para la asistencia y haría más compleja la aprobación del protocolo por el Comité de Ética.

\section{Financiación}

La presente investigación no recibió ayudas específicas provenientes de agencias del sector público, sector comercial o entidades sin ánimo de lucro.

\section{Conflicto de intereses}

Los autores declaran no tener ningún conflicto de intereses.

\section{Bibliografía}

1. Ministerio de Salud Pública, Dirección General de la Salud, División Epidemiología. Primera encuesta nacional de factores de riesgo de enfermedades crónicas no transmisibles, 2006. Anexo IV. Tablas [consultado 22 Ene 2018.]. Disponible 
en: www.msp.gub.uy/sites/default/files/archivos_adjuntos/ anexo_4_tablas_1.pdf

2. Blum-Silva CH, Luz AB, Nascimento MV, et al. Qualitative and quantitative analysis data of the major constituents of Ilex paraguariensis leaves by UPLC-PDA and QTOF-MS. Data Brief. 2016;20:295-9.

3. Heck $\mathrm{Cl}$, de Mejia EG. Yerba Mate Tea (Ilex paraguariensis): A comprehensive review on chemistry, health implications, and technological considerations. J Food Sci. 2007;72:R138-51.

4. Bracesco N, Dell M, Rocha A, et al. Antioxidant activity of a botanical extract preparation of Ilex paraguariensis: Prevention of DNA double-strand breaks in Saccharomyces cerevisiae and human low-density lipoprotein oxidation. J Altern Complement Med. 2003;9:379-87.

5. Bravo L, Mateos R, Sarriá B, et al. Hypocholesterolaemic and antioxidant effects of yerba mate (Ilex paraguariensis) in highcholesterol fed rats. Fitoterapia. 2014;92:219-29.

6. Messina D, Soto C, Méndez A, et al. [Lipid - lowering effect of mate tea intake in dyslipidemic subjects] Spanish. Nutr Hosp. 2015;31:2131-9.

7. Kim SY, Oh MR, Kim MG, et al. Anti-obesity effects of Yerba Mate (Ilex Paraguariensis): A randomized, double-blind, placebo-controlled clinical trial. BMC Complement Altern Med. 2015;15:338.

8. Yu S, Yue SW, Liu Z, et al. Yerba mate (Ilex paraguariensis) improves microcirculation of volunteers with high blood viscosity: A randomized, double-blind, placebo-controlled trial. Exp Gerontol. 2015;62:14-22.

9. Paganini Stein FL, Schmidt B, Furlong EB, et al. Vascular responses to extractable fractions of llex paraguariensis in rats fed standard and high-cholesterol diets. Biol Res Nurs. 2005;7:146-56.

10. Schinella G, Fantinelli JC, Mosca SM. Cardioprotective effects of Ilex paraguariensis extract: Evidence for a nitric oxidedependent mechanism. Clin Nutr. 2005;24:360-6.
11. Branco Cdos S, Scola G, Rodrigues AD, et al. Anticonvulsant, neuroprotective and behavioral effects of organic and conventional yerba mate (Ilex paraguariensis St. Hil.) on pentylenetetrazol-induced seizures in Wistar rats. Brain Res Bull. 2013;92:60-8.

12. Loria D, Barrios E, Zanetti R. Cancer and yerba mate consumption: a review of possible associations. Rev Panam Salud Publica. 2009;25:530-9.

13. Victor BS, Lubetsky M, Greden JF. Somatic manifestations of caffeinism. J Clin Psychiatry. 1981;42:185-8.

14. Freedman ND, Park Y, Abnet CC, et al. Association of coffee drinking with total and cause-specific mortality. N Engl J Med. 2012;366:1891-904.

15. O'Keefe JH, Bhatti SK, Patil HR, et al. Effects of habitual coffee consumption on cardiometabolic disease, cardiovascular health, and all-cause mortality. J Am Coll Cardiol. 2013;62:1043-51.

16. Saito E, Inoue M, Sawada N, et al. Association of coffee intake with total and cause-specific mortality in a Japanese population: The Japan Public Health Center-based Prospective Study. Am J Clin Nutr. 2015;101:1029-37.

17. Newcombe PF, Renton KW, Rautaharju PM, et al. High-dose caffeine and cardiac rate and rhythm in normal subjects. Chest. 1988;94:90-4.

18. Pelchovitz DJ, Goldberger JJ. Caffeine and cardiac arrhythmias: A review of the evidence. Am J Med. 2011;124: 284-9.

19. Cheng M, Hu Z, Lu X, et al. Caffeine intake and atrial fibrillation incidence: Dose response meta-analysis of prospective cohort studies. Can J Cardiol. 2014;30:448-54.

20. Larsson SC, Drca N, Jensen-Urstad M, et al. Coffee consumption is not associated with increased risk of atrial fibrillation: Results from two prospective cohorts and a meta-analysis. BMC Med. 2015;13:207. 